\title{
Synyhesis of Novel Carbazole Based Diol Monomer and Its Photoconducting Polyurethane with Toluene Diisocyanate
}

\author{
Dipak Kumar Mukhopadhyay \\ Institute of Science \& Technology, C.K.Town, West Bengal, 721201, India
}

\begin{abstract}
The novel carbazole based diol monomer has been synthesized by the reaction of $N$-ethyl carbazole3, 6-dicarboxylic acid with propene oxide in the solvent medium of DMF. The novel polyurethane has been synthesized by the reaction of diol monomer with toluene diisocyanate in toluene. The monomer and the polymer have been characterized by IR, UV and NMR spectroscopy. The novel polyurethane has good photoconducting properties. The polymer can be doped with TNF or crystal violet and can be studied dark conductivity and photoconductivity under illumination of different voltages and different intensities. Moveover the polymer can be used to prepare polyurethane foam.
\end{abstract}

Keywords: 3, 6-diacetyl-N-ethyl carbazole, N-ethyl carbazole- 3, 6-dicarboxylic acid, Diol monomer, polyurethane, polyaddition reaction.

\section{Introduction}

Photoconductivity is the increase in electrical conductivity of a material caused by absorption of electromagnetic radiation. Photoconducting polymers are typically very good insulators in dark when the carriers of electricity free electrons and/ or holes are virtually absent. They become more conductive when exposed to light. In order to become a photoconductor, the polymer must satisfy two major requirements:

i. It must absorb light and thus allow photoexcitation of electrons from the ground state. The vacancy left behind ( a hole) and the photoexcited electron from a pair of charge carriers, which can be separated by the action of an electric field.

ii. It must allow migration of either photoexcited electrons or holes, or both, through the polymer in the electric field towards the appropriate electrodes. Since, no ions move and matter is not displaced, the conduction process is wholly electronic with no ionic contribution.

Polymers that do not absorb light of a particular wave length can still support migration of charge carriers generated in an adjacent material. In such a case the polymer are merely as charge transporting media. Since most known organic photoconducting polymers absorb only ultra-violet radiation, extrinsic photogeneration of charges has to be employed to make those polymers active in visible light, and in most practical organic photoconductors, the polymers are only charge-transporting media for charges photogenerated in adjacent photoconductors. Carbazole is another heterocyclic compound that has been used in the synthesis of many organic photoconductors due to its photoconducting properties and high electron donating characters. Therefore these polymers possess interesting electro- optical and photo-chemical properties as well as high thermal stability due to carbazole backbone. Simple carbazole is easily available from coal-tar. Hogel first showed that poly N-vinyl carbazole has efficient photoconducting properties which find applications in devices. A large number of photoconducting polymers have been synthesized using different kinds of polycyclic homo or hetero atomic aromatic compound, but still poly vinyl carbazole is the most important one with respect to efficiency and cost of preparation.

It is known that photon absorption by polymer can generate charge carriers under external electric field and suitable carrier injectors. Polymeric organic photoconductors mostly follow hopping mechanism of conduction. The hopping mechanism is well dependent on structural sequence and regularity of the polymer. The relationship between mechanism of photoionization, photoconduction and trapping of charge carriers with respect to structure is not well established. The photoconducting polymers are finding many important applications like xerography, photoimaging, photorefractive materials and photoelectric diodes.

The aim of this article is to synthesize a novel diol monomer of the following structure:

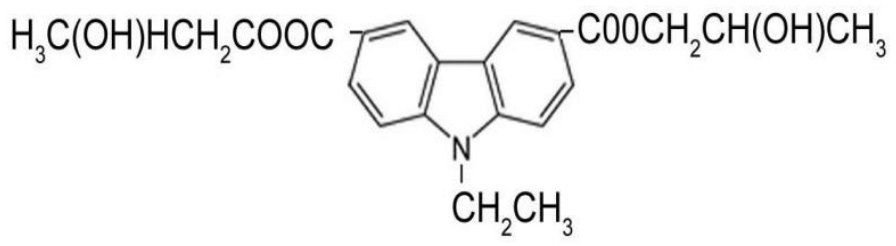

Then preparation of polyurethane of novel diol monomer with toluene di isocyanate. 


\subsection{Synthesis of $\mathrm{N}$-ethyl carbazole:}

\section{Experimental}

To a three-necked round-bottom flask equipped with nitrogen purge and refiux condenser was added $3.34 \mathrm{~g}(0.02)$ of carbazde along with $75 \mathrm{ml}$ of anhydrous DMF. To the stirred solution was added $0.72 \mathrm{~g}$ $(0.03 \mathrm{~mol})$ of sodium hydride. Immediately a precipitate formed with evolution of hydrogen gas. This was stirred for a further 15 minutes to dissolve the carbazole anion. Then $4.68 \mathrm{~g}(0.03 \mathrm{~mol})$ of ethyl iodide was added in one portion. The reaction was exothermic, indicating the reaction proceeded immediately. After $5 \mathrm{~h}, 200 \mathrm{ml}$ of water was added to give a precipitate. The product is recrystallzed from chloroform.

\subsection{Synthesis of 3, 6-Diacetyl-N-ethyl carbazole:}

Finely powdered anhydrous aluminium chloride $(100 \mathrm{~g})$, N-ethyl carbazole $(70 \mathrm{~g})$, and $500 \mathrm{ml}$ dry carbon disulfide were placed in a 1-liter three-necked flask equipped with mechanical stirrer and reflux condenser. $90 \mathrm{~g}$ of acetyl chloride were added dropwise with stirring .The addition generated enough heat to keep the solvent at reflux. The mixture was heated at reflux during an additional hour and the solvent distilled off. $200 \mathrm{ml}$ 1(N) hydrochloric acid and $200 \mathrm{ml}$ ice were added cautiously to the flask. The solid product was removed and washed with water. The product was recrystallized twice from ethyl acetate to give $55 \mathrm{~g}$ of 3,6diacetyl-N-ethyl carbazole. The product was characterized by IR, UV and NMR spectra.

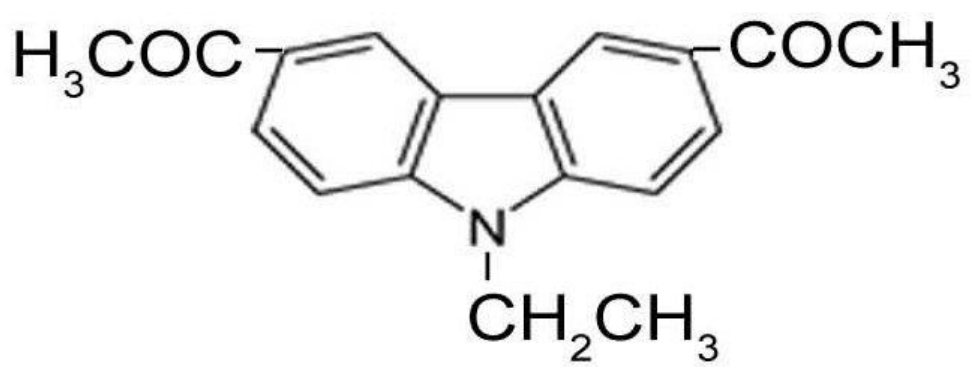

\subsection{Synthesis of N-ethyl carbazole-3, 6-Dicarboxylic acid:}

$9.5 \mathrm{~g}$ of the 3, 6-diacetyl-N-ethyl carbazole was dissolved in $400 \mathrm{ml}$ pyridine and added to $200 \mathrm{ml}$ of 5\% aqueous sodium hydroxide in a 3-liter Erlenmeyer flask. While cooling the mixture in ice, $715 \mathrm{ml}$ of 5\% sodium hypochlorite (bleach) was added. The reaction was allowed to stir $4 \mathrm{~h}$ at room temperature. A concentrated solution of sodium bisulfate was added until no hypochlorite remained (starch-iodine test). The basic solution was neutralized with concentrated hydrochloric acid and the precipitated diacid filtered. The yield of N-ethyl carbazole- 3, 6-dicarboxylic acid was $6.5 \mathrm{~g}$. The product was characterized by IR, UV and NMR spectra.

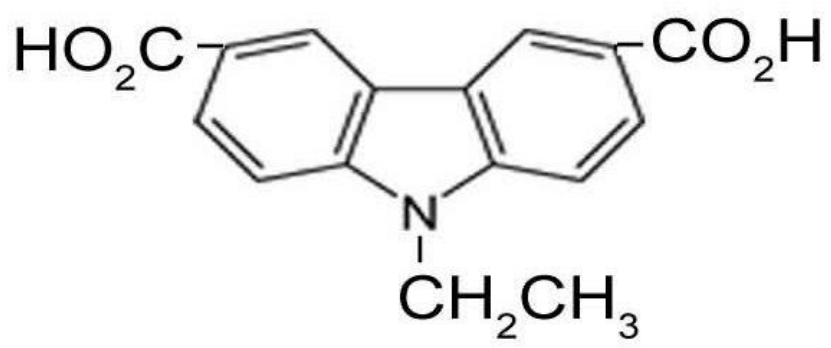

\subsection{Synthesis of novel Diol monomer:}

$11.32 \mathrm{~g}(0.04 \mathrm{~mol}) \mathrm{N}$-ethyl carbazole-3, 6-dicarboxylic acid was added to $100 \mathrm{ml}$ anhydrous DMF. To the stirred solution $4.64 \mathrm{~g}(0.08 \mathrm{~mol})$ propene oxide was added in one portion. The mixture was heated at $60^{\circ} \mathrm{c}$ for $1 \mathrm{~h}$. After completion of the reaction, the mixture was precipitated out in methanol, washed with water and methanol and dried under vacumn. The diol monomer was characterized by IR, UV and NMR spectra.

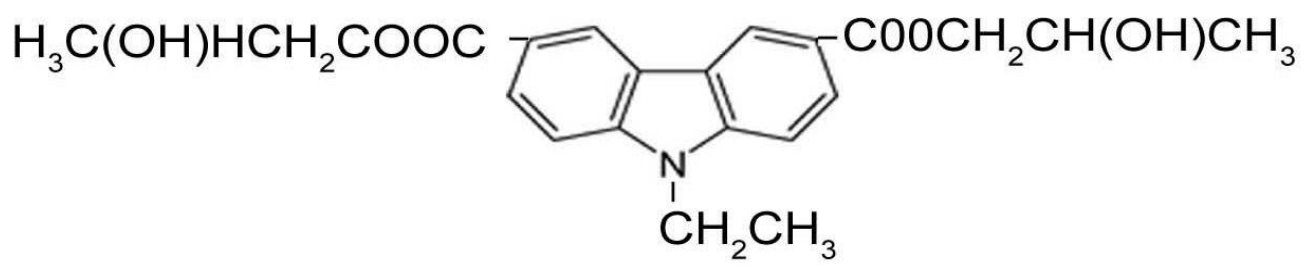


Synyhesis of Novel Carbazole Based Diol Monomer and Its Photoconducting Polyurethane with ..

\subsection{Polymerization:}

0.04 mols diol monomer was dissolved in $150 \mathrm{ml}$ dry toluene. To this stirred solution 0.04 mols of toluene diisocyanate was added and the mixture was stirred vigorously. Then the mixture was warmed at $50^{\circ} \mathrm{c}$ for $1 \mathrm{~h}$. After completion of the reaction, the mixture was precipitated out in methanol, washed with methanol and hexane and dried under vacumn. The polymerization was done by simple polyaddition reaction. The polyurethane was characterized by IR, UV and NMR spectra.

\section{Result and Discussion}

The study of IR, UV and NMR spectra revealed the successful preparation of the polymer. The synthesis of novel diol monomer starting from carbazole is given in scheme-1.

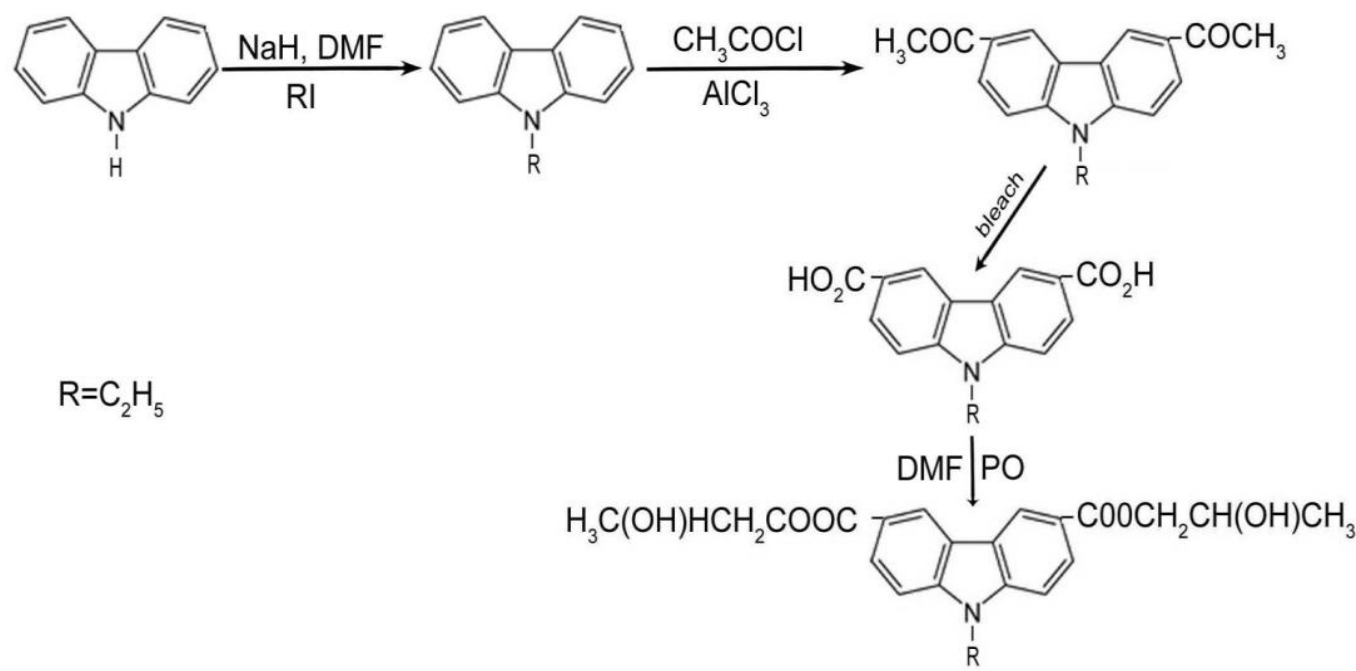

\section{Scheme-1}

N-ethyl carbazole was synthesized by treating carbazole with sodium hydride and ethyl iodide in the solvent medium of DMF. The product was precipitated out in water. 3, 6-diacetyl-N-ethyl carbazole was synthesized by reacting $\mathrm{N}$-ethyl carbazole with acetyl chloride in presence of anhydrous aluminium chloride. After refluxing the mixture the product was precipitated out in ice water. N-ethyl carbazole -3, 6-dicarboxylic acid was synthesized by treating 3, 6-diacetyl- N-ethyl carbazole with sodium hypochlorite in presence of pyridine. The novel carbazole based diol monomer was synthesized by reacting N-ethyl carbazole -3, 6dicarboxylic acid with propene oxide in the solvent medium of DMF. The polyurethane was synthesized by simple polyaddition reaction between the diol monomer and toluene diisocyanate in the solvent medium of toluene. The polymer was precipitated out in methanol. The synthesis route of polymer was given in scheme-II.

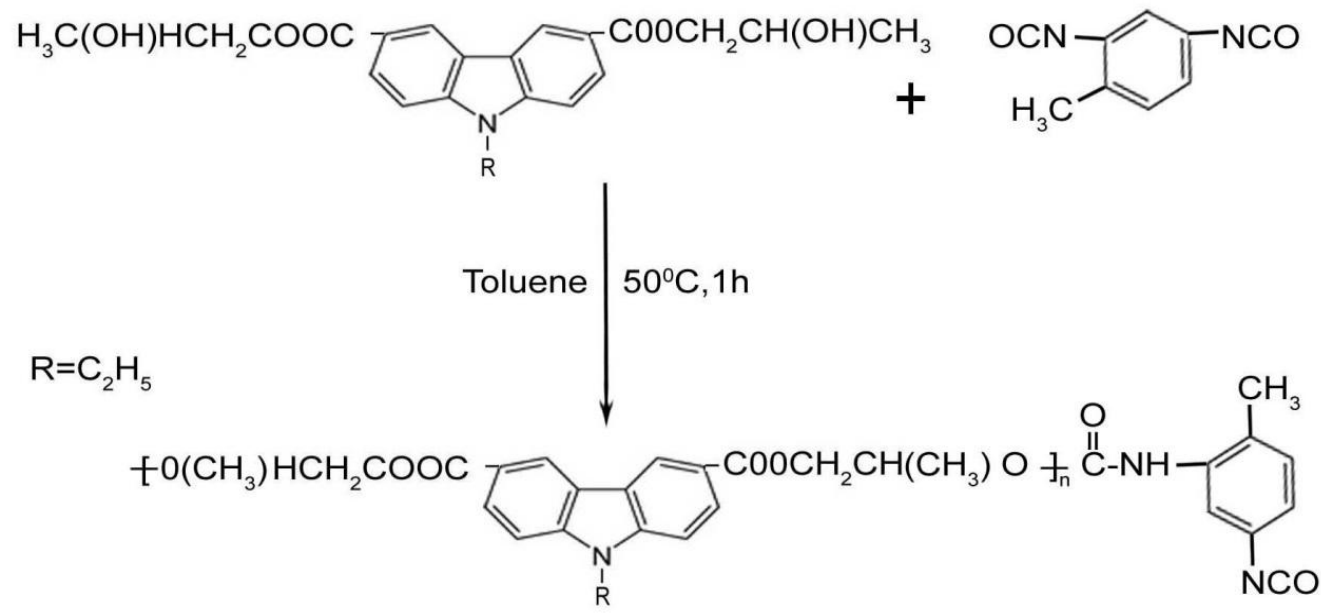


Synyhesis of Novel Carbazole Based Diol Monomer and Its Photoconducting Polyurethane with ..

\section{Scheme-II}

The urethane polymer was soluble in most common organic solvents. The polymer has good mechanical strength and thermal stability due to carbazole backbone. The polymer has good photoconducting property. The polymer can be doped with sensitizer like TNF and crystal violet to enhance the photoconducting property . The doped polymer can be used to measure photoconductivity in the dark or under illumination at different voltages and different intensities. Moreover, the polymer can be used to prepare carbazole based polyurethane foam.

\section{Conclusion}

The article has summarized the synthesis of novel diol monomer and the corresponding polyurethane with the reaction of diol monomer and toluene diisocyanate. The photoconducting polyurethane possess many interesting properties like xerography, electroluminescent property and electrooptical property. The most important polymer sofar in commercial use is poly $\mathrm{N}$-vinyl carbazole. However, this polymer is quite costly and the preparation of the monomer is quite delicate. This investigation will help us to formulate a new and cheaper photoconducting polymer. Moreover, this polymer can be studied for dark conductivity in sandwich configuration at different voltages, different temperatures with different sensitizers like TNF, crystal violet etc. Same studies can be done under illumination at different wavelengths and different intensities.

\section{References}

[1]. H. J. Bolink, C. Arts, V. V. Krasnikov, G. G. Malliaras, G. Hadziioannou, chem. Mater. 9 (1977), 1407.

[2]. W. Huang, W. L. Yu, H. Meng, J. Pei, S. F. Y. Li , chem.. Mater. 10 (1998), 3340.

[3]. A. C. Arango, S. A. Carter, P. J. Brock, Appl. Phys. Lett. 74 (1999), 1698.

[4]. O. Ostroverkhova, W. E. Moerner, chem. Rev. 104 (2004), 3267.

[5]. T. K. Daubler, L. Kulikovsky, D. Neher, V. Cimrova, J. Hummelen, E. Mecher, R. Bittner, K. Meerholz, Proc. Of SPIE 4462 (2002), 206.

[6]. J. Grazulevicius, P. Strohriegl, J. Pielichowski, K. Pielichowski, Prog. Polym. Sci. 28 (2003), 1297

[7]. Y. Zhang, H. Sasabea, J. Mater. Chem. 8 (1998), 809.

[8]. E. Mecher, C. Brauchle, H. H. Horhold, J. C. Hummelenc, K. Meerholz, Phys.Chem.Chem.Phys. 1 (1999), 1749.

[9]. M.S. Bratcher, M. S. DeClue, A. Grunnet- Jepsen, D. Wright, B. R. Smith, W. E. Moerner, J. S. Siegel, J. Am.Chem.Soc. 120 (1998), 9680.

[10]. D. Yang, L. Li, C. Wang, Mater. Chem. Phys. 87 (2004), 114.

[11]. S. Shim, M. Suh, S. Suh, X. L. Huang, E. Suh, Polymer 41 (2000), 467.

[12]. V. C. Kishore, R. Dhanya, C. S. Kartha, K. Sreekumar, R. Joseph, J. Appl. Phys. 101 (2007), 0631021.

[13]. S. C. Suh, S. C. Shim, Synth. Met. 114 (2000), 91.

[14]. S.M.Teleb, A. S. Goballa, Spectrochim. Acta. Part A 62 (2005) 140.

[15]. H. Chun, I. K. Moon, D. H. Shin, N. Kim, Chem. Mater. 13 (2001) 2813.

[16]. W.G.Jun, M. J. Cho.D. H. Choi, J. Korean, Phys.Soc. 47 (2005)620.

[17]. L. Angiolini, T. Benelli, L. Giorgini, E. Salatelli, Polymer 46 (2005) 2424.

[18]. M. Toba, Y. Takeoka, M. Rikukawa, K. Sanui, Synth. Met. 152 (2005) 197.

[19]. F. C. Cebeci, E. Sezer, A. S. Sarac, Electrochim. Acta. 52 (2007) 2158

[20]. L.Angiolini, D.Caretti, 1. Giorgini, E. Salatelli, Polymer 42 (2001) 4005. 